\title{
Auditoria em Enfermagem: identificando sua concepção e métodos*
}

\author{
Nursing Audit: identifying notions and method \\ Auditoría de Enfermería: identificando las concepciones y método
}

Ariane Fazzolo Scarparo', Clarice Aparecida Ferraz'
'Universidade de São Paulo, Escola de Enfermagem de Ribeirão Preto,
Departamento de Enfermagem Geral e Especializada. Ribeirão Preto, SP

Submissão: 26/06/2007

Aprovação: 01/12/2007

\section{RESUMO}

O presente estudo objetivou identificar e analisar opiniões de especialistas em auditoria Que atuam no contexto da enfermagem e sistematizar tendências de concepção, método e finalidade da auditoria de enfermagem, na atualidade e para os próximos cinco anos. Metodologicamente, a investigação foi estruturada utilizando-se da técnica Delphi, tipo de avaliação prospectiva e consensual de tendências, por parte de especialistas no tema investigado. Os resultados revelaram Que a concepção atual da auditoria está enfocada na visão contábil e financeira, tendo em vista a sustentação econômica do hospital e como ato de controladoria visando identificar pagamentos indevidos referentes à conta hospitalar; no futuro, a essa concepção será associada à avaliação da Qualidade da assistência, com envolvimento em outras áreas Que nela interferem.

Descritores: Auditoria de enfermagem; Economia; Qualidade da assistência à saúde.

\section{ABSTRACT}

The present study aimed at identifying and analyzing the opinions of auditing experts who work within the context of nursing and systematizing trends regarding the notion, methods and purposes of nursing audits at present and over the next five years. In terms of methodology, the study was structured using the Delphi technieue, type of prospective and consensual evaluation of trends, performed by experts on the theme under investigation. Results demonstrated that the current notion of nursing audits focuses on the accounting and financial elements, the financial maintenance of the hospital being kept in mind as well as the controlling activity of trying to identify incorrect hospital bills. In the future, however, the notion of auditing is expected to become associated with evaluating the Quality of care, with the involvement of other areas that have an impact on it.

Descriptors: Nursing audit; Economics; Quality of health care.

\section{RESUMEN}

La presente investigación teve como objetivos identificar y analizar opiniones de especialistas en auditoría Que actúan en el contexto de la enfermería y sistematizar tendencias de concepción, de método y de finalidad de la auditoría en enfermería, en la actualidad y para los próximos cinco años. Metodológicamente, la investigación fue estructurada utilizando la técnica Delphi, tipo de evaluación prospectiva y consensual de tendencias, por parte de especialistas en el tema investigado.Los resultados revelaron Que la concepción actual de la auditoría se concentra en la visión contable y financiera, teniendo en vista la sustentación económica del hospital y como acto de contraloría a los efectos de identificar pagos indebidos referentes a la cuenta hospitalaria; en el futuro, esa concepción referida será asociada a la evaluación de la calidad de la asistencia, con participación en otras áreas Que en ella interfieren.

Descriptores: Auditoría de enfermería; Economía; Calidad de la atención de salud.

*Extraído da dissertação de mestrado apresentada à Escola de Enfermagem de Ribeirão Preto da Universidade de São Paulo, em 2007. 


\section{APRESENTAÇÃO DO PROBLEMA DE INVESTIGAÇÃO}

Meu primeiro contato com a auditoria na área da saúde deu-se em um hospital geral de grande porte, na cidade de São Paulo. A área contábil e financeira apresentou-se, então, a mim como atividade desconhecida, o Que despertou meu interesse para a realização de um curso de auditoria de contas hospitalares

No trabalho de auditoria, observei pouca preocupação formal com a avaliação do cuidado prestado ao paciente, situação esta Que me causava inquietação, pois era bastante pertinente a oportunidade de realizá-la concomitantemente à análise contábil. Pude também categorizar erros mais freeüentes Que geravam glosas na conta hospitalar, constatando, assim, Que a maioria era de responsabilidade da enfermagem, o Que me incentivou a sistematizar algumas condutas nesta área.

É interessante ressaltar Que, após o envolvimento de toda a equipe, o índice de glosas que era de $15 \%$ foi reduzido para 3\% do faturamento do hospital. Porém, a avaliação da Qualidade da assistência de enfermagem não foi sistematicamente estruturada. Nesse momento, a Questão da auditoria Qualitativa emergia como um problema a ser estudado.

Diante desta constatação, notei a necessidade de se clarear a concepção, os métodos, a finalidade e os parâmetros legais Que sustentam, na prática, a auditoria em enfermagem, contribuindo, assim, para a sistematização do trabalho do enfermeiro auditor.

$\mathrm{Na}$ área da saúde, a auditoria surge pela primeira vez no estudo realizado pelo médico George Gray Ward, nos Estados Unidos, em 1918, no Qual foi verificada a Qualidade da assistência médica prestada ao paciente por meio dos registros em prontuário( ${ }^{(1)}$. Assim, o princípio da auditoria na área da saúde focalizou-se na avaliação da Qualidade assistencial prestada ao paciente, visto Que esta é o cerne para a prática dos profissionais desta área. No entanto, atualmente, com o aumento da competitividade entre serviços hospitalares, passou-se a oferecer tratamentos mais dispendiosos financeiramente, ressaltando a preocupação em otimizar seus custos. Isso passou a reQuerer a atuação de profissionais capacitados nesta área, o Que exigiu também uma visão econômico/contábil de forma a operacionalizar o processo de auditoria. Desta forma, ela incorporou-se à rotina das instituições de saúde com o intuito de avaliar os aspectos Qualitativos da assistência requerida pelo paciente, os processos internos e as contas hospitalares.

Atualmente há um grande número de hospitais, predominantemente privados, Que possuem serviços de auditoria Que contam com a atuação de profissionais da área da saúde. Alguns, segundo a literatura, criaram comissões de auditoria para avaliar a organização em áreas internas de controle, contas e finanças, fortalecendo a estrutura em épocas de dificuldades financeiras para os hospitais ${ }^{(2)}$. No entanto, no Brasil, criou-se a idéia equivocada de que a auditoria em saúde é aquela relacionada a atividades estritamente burocráticas, de cunho contábil e financeiro.

Os serviços públicos também se adeQuaram a esta realidade, segundo legislação Que normatiza o acompanhamento fiscal, o controle e a avaliação técnico-científica, contábil, financeira e patrimonial das ações e serviços de saúde. Em 1999, o Ministério da Saúde reestruturou a nova organização de atividades do SNA, sendo Que aQuelas pertinentes ao controle e avaliação passaram à responsabilidade da Secretaria de Assistência à Saúde (SAS) e, as referentes à auditoria, ao Departamento Nacional de Auditoria do SUS (DENASUS) ${ }^{(3)}$.

A auditoria em enfermagem representa a função de controle do processo administrativo, verificando se os resultados da assistência estão de acordo com os objetivos ${ }^{(4)}$. No entanto, há autores Que a definem como a avaliação sistemática da Qualidade de enfermagem prestada ao cliente pela análise dos prontuários, garantindo justa cobrança e pagamento adeQuado ${ }^{(5)}$.

Com a padronização dos processos da assistência de enfermagem, a avaliação a ser realizada por meio da auditoria passa a ter um suporte de forma Que a prática assistencial tenha condições de avaliar seus resultados.

Para o exercício da auditoria é importante a construção de instrumentos Que devem contemplar os objetivos de acordo com a estrutura, processo e resultado da assistência, definindo o caminho a percorrer para o alcance da Qualidade ${ }^{(6)}$.

Mesmo com a apreensão desses conhecimentos, a revisão da literatura permite observar Que as dimensões conceitual, de métodos, de finalidade e legislação da auditoria em enfermagem permanecem com lacunas de compreensão, reQuerendo novas investigações.

Assim, o presente estudo teve como objetivos identificar e analisar opiniões de especialistas em auditoria Que atuam no contexto da enfermagem brasileira e sistematizar tendências de concepção, método e finalidade da auditoria em enfermagem na atualidade e para os próximos cinco anos.

\section{METODOLOGIA}

\section{Utilização da Técnica Delphi}

A investigação foi estruturada utilizando-se a técnica Delphi, Que consiste em um tipo de avaliação prospectiva e consensual de tendências, por parte de especialistas no tema investigado. Tal técnica é indicada Quando há inexistência de dados, necessidade de abordagem multidisciplinar ou mesmo Quando há falta de consenso em determinado assunto.

As características essenciais da técnica Delphi são o anonimato, a retroalimentação das respostas, análise estatística dos dados em cada fase do estudo e interação para a obtenção do consenso ${ }^{(7)}$.

Não existe um número ideal de participantes estabelecido para a composição do grupo. Para a definição dos peritos é de suma importância seu grau de especialização, a avaliação do tema, os fatores de custo e a natureza do problema; o número de peritos disponível deve ser destacado(8).

Trata-se de um Questionário, Que circula diversas vezes (denominado rodadas), pelo grupo de especialistas, preservando o anonimato das respostas. Na primeira rodada os especialistas, também denominados painelistas ou juizes, recebem um Questionário, Quando são solicitados a responder com respostas Quantitativas apoiadas por justificativas e informações Qualitativas, sendo realizado feedback de respostas do grupo para as rodadas subseQüentes ${ }^{(9)}$.

Assim, repetem-se as perguntas em várias rodadas e os participantes reavaliam suas respostas com base nas justificativas dadas pelos outros participantes nas rodadas anteriores, até que a divergência das opiniões reduza-se a um nível em Que as respostas da última rodada sejam consideradas a previsão do grupo ${ }^{(9)}$. 
O primeiro passo para a implementação da técnica foi identificar os especialistas sobre a temática a ser estudada, compondo, assim, o grupo de juizes ou painelistas que opinariam sobre o assunto

\section{InQuérito sobre Auditoria de Enfermagem}

Tendo em vista a elaboração do Questionário de coleta de dados o pesquisador recorreu à literatura especializada a fim de indagar o conhecimento produzido construindo um ineuérito acerca do tem. Assim procedeu-se o esquadrinhamento do conhecimento produzido e divulgado em periódicos científicos indexados nos bancos de dados: Literatura Latino-Americana e do Caribe em Ciências da Saúde (LILACS), Literatura Internacional em Ciências da Saúde (Medline) e Banco de Dados Bibliográficos da Universidade de São Paulo Catálogo on-line global (Dedalus), nos últimos 10 anos (1996 a 2005), Que tratavam das dimensões da Auditoria de Enfermagem em investigação.

$\mathrm{Na}$ base de dados LILACS foi realizado o levantamento da bibliografia a Qual continha, em seu resumo, o cruzamento entre as palavras-chave auditoria e enfermagem, sendo analisados 10 artigos desta base. Ainda no Lilacs foi realizado o cruzamento das palavras auditoria e hospital, sendo utilizados três desses estudos.

No Dedalus, do cruzamento de auditoria e enfermagem, em todos os campos e bases, foram utilizados três estudos. No Medline, foi selecionado o artigo científico Que continha em seu título o cruzamento entre as palavras auditoria e enfermagem, sendo que três estudos foram pertinentes a esta pesQuisa.

Ao final da análise bibliográfica foram selecionados 19 artigos, dos Quais se excertou as informações referentes à concepção, método, finalidade e referência legal da auditoria em enfermagem. Estes dados estão referenciados em quadros, no estudo original.

A análise destes estudos demonstra Que a definição de auditoria em enfermagem ou em saúde segundo 64,3\% dos autores está relacionada à análise Qualitativa da assistência de enfermagem prestada, corroborando com a premissa do trabalho do enfermeiro de oferecer ao seu cliente assistência de boa Qualidade. Embora $21,4 \%$ a relacionem à avaliação contábil, tem-se como pressuposto Que, na prática atual, o enfoque das instituições de saúde, é de vincular a auditoria à Questões contábeis-financeiras.

Quanto aos métodos de trabalho relatados pelos autores estudados, poucos autores explicitaram claramente o método utilizado, não havendo seguimento de padrões. A auditoria de enfermagem pode ser realizada com uma finalidade ou várias outras agregadas.

\section{Coleta de Dados}

Baseando-se na técnica Delphi, o instrumento de coleta de dados elaborado contém Questões de tendências e de prioridades sobre as dimensões de concepção, métodos e finalidades da auditoria de enfermagem.

O projeto foi submetido à apreciação do Comitê de Ética em Pesquisa da Escola de Enfermagem de Ribeirão Preto da Universidade de São Paulo, obtendo aprovação para sua realização.

Os participantes foram selecionados por meio de contatos via internet, por meio de sites de instituições de saúde. Participaram 198 enfermeiros, docentes da área de administração em enfermagem das universidades públicas e privadas brasileiras, enfermeiros auditores de instituições públicas, privadas $\mathrm{e}$ operadoras de saúde e enfermeiros atuantes em áreas gerenciais.

Foi realizado contato via correio eletrônico e correio convencional, enviando a Apresentação da Pesquisa aos Participantes Selecionados e convite para a participação. Destes, 78 enfermeiros responderam positivamente ao convite de colaborar com a pesQuisa.

A coleta de dados foi realizada em duas rodadas, conforme recomendado. Na primeira etapa, os especialistas, também chamados de painelistas ou juízes, receberam o instrumento de peseuisa em formato de Questionário, suas respostas foram organizadas e analisadas buscando identificar convergências entre os participantes.

O instrumento e o termo de consentimento livre e esclarecido foram enviados para 78 participantes dando início à primeira rodada, a Qual se encerrou com 54 instrumentos respondidos. Após a análise dos dados obtidos na primeira rodada, o instrumento foi, então, reformulado, sendo retirados os cinco sub itens nos se obteve consenso de $70 \%$ ou mais nas respostas. Este segundo instrumento foi enviado aos painelistas, dando início a segunda rodada. Nessa, obteve-se 34 instrumentos respondidos.

Os dados foram sistematizados segundo os Quesitos da auditoria de enfermagem investigados, sendo digitados e armazenados em banco de dados estruturado no Microsoft Excel.

\section{RESULTADOS E DISCUSSÃO}

Os resultados serão apresentados segundo estatística descritiva, considerando os objetivos da pesquisa e a matriz de quesitos que compõem o instrumento de coleta de dados.

A princípio, será apresentada a caracterização dos sujeitos da amostra e, na seqüência, as tendências identificadas nas rodadas no Que se refere à concepção, métodos e finalidades da auditoria em enfermagem e, por último, as prioridades consideradas pelos painelistas Que deverão ser implementadas no futuro.

Os participantes foram enfermeiros Que atuam na docência $(41,2 \%)$, em serviços de saúde $(52,9 \%)$, sendo que $(5,9 \%)$ atuam nas duas áreas. Quanto à experiência na área de administração em enfermagem, 67,6\% dos participantes possuem três anos ou mais. No entanto, nota-se que, embora $64,8 \%$ possuam experiência em auditoria em enfermagem, desses, 35,3\% têm três anos ou mais de experiência nesta área.

Com relação à pós-graduação, 51 ,2\% a possuem na área de administração em enfermagem, sendo Que há participantes Que possuem mais de um tipo de formação. Quanto à especialização ou pós-graduação em auditoria em enfermagem, 84,4\% dos painelistas não possuem nenhuma formação específica nesta área.

Houve consenso no Que diz respeito à concepção da auditoria em enfermagem, segundo visão burocrática de cunho contábil e financeiro, tendo em vista a sustentação econômica do hospital, da seguinte forma: muito provável Que esteja ocorrendo na atualidade $(79,4 \%)$ e que esse enfoque venha a ser implementado nos próximos cinco anos (85,3\%).

A auditoria em enfermagem, concebida como exame sistemático e independente para avaliar se as atividades de enfermagem são implementadas de acordo com o planejamento da assistência de enfermagem, visando alcançar objetivos e resultados, foi considerada pouco provável na atualidade $(70,6 \%)$ e muito provável nos próximos cinco anos $(79,4 \%)$. 
A concepção da auditoria em enfermagem, segundo as vertentes da Qualidade e contábil, a fim de avaliar, de forma abrangente, a gestão da assistência de enfermagem, obteve consenso (82,3\%) apenas na projeção para os próximos cinco anos. O enfoQue para a atualidade não chegou ao consenso estabelecido.

Em relação ao futuro (próximos cinco anos), os participantes consideram Que a concepção da auditoria em enfermagem estará pautada da seguinte forma:

- segundo as vertentes da Qualidade e contábil, a fim de avaliar, de forma abrangente, a gestão da assistência de enfermagem; - com envolvimento em outras áreas Que interferem, direta ou indiretamente, na assistência de enfermagem;

- segundo visão científica e técnica da assistência visando a Qualidade do cuidado;

- segundo visão burocrática, de cunho contábil e financeiro, tendo em vista a sustentação econômica do hospital,

- como exame sistemático e independente para avaliar se as atividades de enfermagem são implementadas de acordo com o planejamento da assistência de enfermagem, visando, assim, alcançar objetivos e resultando;

- como ato de controladoria hospitalar, visando identificar pagamentos indevidos de contas hospitalares;

- segundo visão da melhoria da Qualidade, buscando identificar inconformidades dos processos a partir de padrões estabelecidos para a assistência de enfermagem.

Com relação ao método utilizado para a execução da auditoria em enfermagem, obteve-se consenso como sendo muito provável a utilização da análise retrospectiva na atualidade $(79,4 \%)$ e nos próximos cinco anos $(88,3 \%)$.

De modo geral, os resultados revelaram Que a concepção atual da auditoria está enfocada na visão contábil e financeira, tendo em vista a sustentação econômica do hospital e como ato de controladoria, visando identificar pagamentos indevidos referentes à conta hospitalar; no futuro, a essa concepção referida será associada à avaliação da Qualidade da assistência, com envolvimento em outras áreas Que nela interferem. Os métodos e finalidades Que obtiveram consenso entre os participantes para a atualidade e futuro estão intimamente relacionados à concepção de tais períodos. Na atualidade, o método da auditoria em enfermagem foi considerado de forma retrospectiva, do tipo interna, contemplando as etapas de coleta de dados e análise de contas hospitalares a fim de impor glosas ou diminuí-las, utilizando dados coletados dos prontuários dos pacientes, registros e manuais de enfermagem.

No futuro, foram associados a esses métodos, a forma concorrente, o tipo externa, contemplando a análise da estrutura, processo e resultado, considerando visão integrada e ampliada, a definição de objetivos e a coleta de dados acerca dos processos da assistência de enfermagem com elaboração de relatório técnico.

A finalidade da auditoria em enfermagem, na atualidade, está restrita à comprovação de pagamento de contas hospitalares, revendo glosas por meio do relatório técnico e realizando negociações entre representantes do hospital e do convênio; no futuro, associado à primeira finalidade a intenção será apontar inadeQuações da assistência de enfermagem, reformulando suas práticas, indicando processos de educação em serviço e delineando ações corretivas. Com relação às prioridades a serem implementadas para a prática futura foram pontuados a formação específica (especialização) e o planejamento e execução da assistência de forma sistemática, pautada no conhecimento científico e técnico da profissão.

Conclui-se Que a auditoria em enfermagem cumpre uma finalidade institucional Que, na atualidade, está pautada em um enfoque empresarial e mercadológico. Há uma tendência de mudança do enfoeue de mercado voltado para o cliente, portanto, pautado na Qualidade do produto ou serviço, havendo a adeQuação das ações da auditoria em enfermagem nesse sentido.

\section{REFERÊNCIAS}

I. Kurcgant P. Auditoria em enfermagem. Rev Bras Enferm 1976; 29: 106-24.

2. Urbani FR, Hauser RC. Hospital audit commitees: a comparative analysis of structural and functional characteristics. Hospital \& Health Serv Adm 1991; 36(3): 383-96.

3. Ministério da Saúde (BR). Departamento Nacional de Auditoria do SUS. Brasília (DF). Disponível em: URL: http:// www.saude.gov.br

4. Silva SH, Ortiz DCF, Shimizu HE, Toth M. Auditoria em enfermagem: implantação e desenvolvimento no hospital universitário da Universidade de São Paulo. Rev Esc Enferm USP 1990; 24(2): 199-209.

5. Motta ALC. Auditoria de enfermagem nos hospitais e operadoras de planos de saúde. I a ed. São Paulo (SP): látria; 2003.

6. Cianciarullo TI. Teoria e prática em auditoria de cuidados. São Paulo (SP): Ícone; 1997.

7. Dal Ben LW. Instrumento para dimensionar horas diárias de assistência de enfermagem residencial [dissertação]. São Paulo (SP): Escola de Enfermagem, Universidade de São Paulo; 2000.

8. Spinola AWP. Técnica prospectiva delfos abordagem teóricoprática. São Paulo (SP): SM Gráfica e Editora Ltda; 1997.

9. Wright ITC, Giovinazzo RA. Delphi - uma ferramenta de apoio ao planejamento prospectivo. Cad Pese Adm 2000; I(12): 54-65. 